\title{
Relationships among Disability, Quality of Life, and Physical Fitness in Lumbar Spinal Stenosis: An Investigation of Elderly Korean Women
}

\author{
Saejong Park ${ }^{1}$, Ho Sung Han ${ }^{2}$, Gang-Un Kim ${ }^{3}$, Sung Shik Kang ${ }^{3}$, Ho-Joong Kim³ , \\ Mihyun Lee ${ }^{1}$, Soo Hyun Park ${ }^{1}$, Kyu Hwan $\mathrm{Choi}^{4}$, Sung-Ho Kim ${ }^{5}$, Jin S. Yeom ${ }^{3}$ \\ ${ }^{1}$ Department of Sports Science, Korea Institute of Sports Science, Seoul, Korea \\ ${ }^{2}$ Department of Orthopaedic Surgery, Spine Center, National Medical Center, Seoul, Korea \\ ${ }^{3}$ Department of Orthopaedic Surgery, Spine Center, Seoul National University Bundang Hospital, \\ Seoul National University College of Medicine, Seongnam, Korea \\ ${ }^{4}$ Department of Sports Science, Sungkyunkwan University, Suwon, Korea \\ ${ }^{5}$ Department of Sports Medicine, Kyung Hee University, Yongin, Korea
}

\section{Study Design: A cross-sectional, case-control study.}

Purpose: To investigate associations between physical fitness measures and disabilities related to back pain and quality of life (0OL) by the presence of symptomatic lumbar spinal stenosis (LSS) in elderly Korean women.

Overview of Literature: LSS leads to decreased functioning and reduced QOL. However, correlations among physical fitness, disability, and QOL have not been investigated in elderly women with LSS.

Methods: Participants included women aged 65 years and older ( $n=192)$, divided into a study group ( $n=38$ ) and a control group ( $n=154)$ based on the presence/absence of LSS. All participants underwent physical function and fitness tests. Oswestry disability index (ODI) scores and EuroQol five-dimensional questionnaire (EQ-5D-5L) scores were used to assess disability and health-related QOL.

Results: The results for the handgrip strength, sit-and-reach, functional reach, and timed up and go (TUG) tests were significantly higher in the control group than the LSS group. ODI scores were significantly higher and EQ-5D-5L scores significantly lower in the LSS group. TUG and functional reach test scores were significantly correlated with ODI scores, and handgrip strength was strongly interrelated with ODI and EQ-5D-5L scores in the LSS group. No other physical fitness measures showed statistically significant relationships with ODI or EQ-5D-5L scores.

Conclusions: In elderly Korean women with LSS, back pain-related disability and QOL are significantly associated with some physical fitness parameters such as handgrip strength. Handgrip strength reflects general muscle strength, which is significantly interrelated with the level of disability and QOL. Our results suggest that enhancing generalized muscle strength helps to reduce disability due to back pain and improve $00 \mathrm{~L}$ in patients with LSS.

Keywords: Lumbar vertebrae; Spinal stenosis; Physical fitness; Oswestry disability index; Euro0ol five-dimensional questionnaire

Received Mar 21, 2016; Revised Jul 29, 2016; Accepted Aug 5, 2016

Corresponding author: Ho Sung Han

Department of Orthopaedic Surgery, Spine Center, National Medical Center,

245 Eulji-ro, Jung-gu, Seoul 04564, Korea

Tel: +82-2-2276-2145, Fax: +82-2-2267-8685, E-mail: tongnam4@gmail.com 


\section{Introduction}

Lumbar spinal stenosis (LSS) is a degenerative spinal disease that results in progressive narrowing of the spinal canal, leading to compression of the nerve root $[1,2]$. LSS is a common leading cause of physical inactivity and functional impairment in older adults, which is mostly attributed to leg and lower back pain and neurogenic claudication. LSS considerably reduces an individual's quality of life (QOL) [3]. Moreover, various factors (e.g., comorbidities and demographic factors) are collectively associated with LSS-related functional impairment and physical inactivity [4]. Therefore, it is important to clarify the factors associated with physical inactivity and functional impairment in patients with LSS.

Physical fitness represents a person's general physical health status [5]. The level of physical fitness has been associated with age-associated diseases such as hypertension, diabetes, and cognitive impairment [6-8]. Furthermore, some physical fitness parameters have been associated with QOL and severity of pain among patients with musculoskeletal diseases [5]. Although the precise mechanisms are unknown, pain sensitization and proinflammatory cytokines are believed to be involved $[9,10]$.

As LSS is a major cause of physical inactivity and functional impairment, patients with LSS often experience a decline in physical fitness. Decreased physical fitness can be a common factor leading to pain in older adults. Moreover, decreased physical fitness contributes to decline in QOL and the activities of daily living. Therefore, a strong interrelationship may exist between physical fitness and LSS. However, few studies have investigated these associations. We investigated the association between the degree of disability from back pain, QOL, and physical fitness parameters in women with LSS aged over 65 years.

\section{Materials and Methods}

\section{Study design and participants}

We designed this cross-sectional study as part of a national program to develop criterion-referenced health-related fitness standards for the National Fitness Award, conducted between March and November 2014. The study was approved by the Institutional Review Board of the corresponding author's organization (IRB no. B-1405/252-005). Written informed consent was obtained from all partici- pants before enrollment in the study. The study included two groups: a study group (LSS group), consisting of symptomatic patients with LSS who visited the spine center at the last author's hospital, and a control group, comprising randomly chosen women from six cities in Korea. Inclusion criteria for the LSS group were (1) women aged between 65 and 85 years, (2) stenotic lesion in the lumbar spine confirmed via radiology, and (3) symptoms (one or more) of gluteal/lower extremity pain or numbness exacerbated by walking or standing, which was improved or resolved by sitting or bending forward, motor function deficits in the lower extremities and buttocks, and bladder/bowel dysfunction thought to be caused by spinal stenosis [11]. Radiological stenotic lesions were confirmed with lumbar magnetic resonance imaging [12]. Inclusion criteria for the control group were (1) women aged between 65 and 85 years who were (2) generally healthy and could perform physical fitness tests. We excluded participants who were unable to perform physical fitness tests because of pain in the knee, hip, or ankle joint, or a serious medical condition, and participants who were unable to complete the questionnaires about pain severity and disability. Study group participants were enrolled at the spine center of a tertiary-care teaching institution. Control group participants were randomly selected from six cities for the abovementioned national program, and enrolled through National Fitness Award program centers or senior citizen community centers. All participants in this study were enrolled between May and November 2014. In total, 38 women with a diagnosis of LSS were defined as the LSS group and 154 healthy women were defined as the control group.

\section{Measurements}

Measurements used to assess the relationships among different parameters were taken after confirmation of inclusion and exclusion criteria. All data were collected by blinded clinical research assistants. Anthropometric data were collected for comparison of basic characteristics between the two groups. Height and weight were measured using standard methods. Body mass index (BMI) was calculated as weight divided by the square of height $\left(\mathrm{kg} / \mathrm{m}^{2}\right)$. Body composition, including fat content (\%) and skeletal muscle mass $(\mathrm{kg})$, was measured with bioimpedance analysis using InBody 720 (Inbody Corp., Seoul, Korea), a tool approved by the US Food and Drug Administration 
and validated by research [13]. Waist circumference was measured at the navel and recorded to the nearest centimeter. Eight standardized physical fitness tests, including handgrip strength, cross-arm crunch sit ups, trunk flexion-sit and reach, prone trunk extension, prone isometric chest raise, sit-to-stand (STS) test, functional reach, and the timed up and go (TUG) test, were performed for comparison of physical fitness in the two groups. The results of these tests were the primary outcomes of this investigation. Disability and QOL were evaluated using self-completed questionnaires: the Oswestry disability index (ODI), previously validated in Korea [14], to assess functional disability related to lower back pain, and the Korean version of the EuroQol five-dimensional questionnaire (EQ5D-5L), to evaluate health-related QOL. ODI and EQ-5D$5 \mathrm{~L}$ results were the secondary outcomes of this study.

\section{Physical performance tests}

All testing sessions were conducted by experienced standardized researchers.

1) Handgrip strength (generalized muscle strength) Muscular strength was assessed by a handgrip strength test [15]. Handgrip strength $(\mathrm{kg})$ was measured with a digital dynamometer (GRIP-D 5101, Takei, Tokyo, Japan). Measurements were performed twice for both hands, and the mean values were recorded.

\section{2) Cross-arm sit-up test (muscle endurance)}

Muscle endurance was assessed with a cross-arm sit-up test [15]. Participants were instructed to fix their feet on the floor with both arms crossed over their chest. Starting in a supine position, they were instructed to sit up repeatedly while maintaining the position of their feet and arms for 60 seconds. The number of repetitions in 60 seconds was recorded (repetitions/60 sec).

\section{3) Sit-and-reach test (flexibility)}

Participants' general flexibility was assessed with a sitand-reach test [16]. The test was performed with participants in a sitting position on a flat floor with their legs fully extended. Participants' feet were placed against the base of the sit-and-reach box, with their toes pointing up. They were asked to place one hand over the top of the other hand and bend slowly forward as far as possible, sliding both hands on the measuring board while keeping both knees straight, and hold the position for at least 2 seconds. The distance reached was recorded.

4) Prone trunk extension (back extensor muscle strength and flexibility)

For the prone trunk extension and chest raise test, participants were instructed to lie flat on the floor in a prone position with both hands under their thighs [17]. They were then asked to lift their upper body from the floor slowly from the head, arms, and chest, as high as possible. The highest point that the participants' chin reached $(\mathrm{cm})$ was recorded.

5) Prone isometric chest raise test (back extensor endurance) The back-muscle endurance of each participant was assessed with the prone isometric chest raise test [17]. Participants were placed prone on the floor and instructed to raise their upper body by $30^{\circ}$, lifting their sternum from the floor. They were asked to hold this position for as long as possible, and the total endurance time(s) was recorded.

6) STS test (functional mobility, strength of the lower extremities)

For the STS, participants were asked to sit in the middle of a standard chair $(43 \mathrm{~cm})$ without arm rests and with a straight back and crossing both arms over the chest [18]. Then, they were instructed to rise to a standing position and return to the sitting position repeatedly as fast as possible for 30 seconds. The total number of times a participant came to a full standing position within 30 seconds was recorded (repetitions/30 sec).

\section{7) Functional reach test (stability)}

For the functional reach test, a yardstick was prepared and attached to a flat wall at approximately shoulder height. Participants were instructed to stand close to the wall and raise their arms with a closed fist and a shoulder flexion of $90^{\circ}$ [19]. The examiner recorded the position of the third metacarpal head on the yardstick. Next, participants were instructed to reach their arms forward as far as possible without moving their feet. The location of the third metacarpal head on the yardstick was recorded, and the difference between the start and end locations was recorded as the functional reach distance $(\mathrm{cm})$.

8) TUG test (functional mobility and balance) 
The TUG test was performed to assess participants' mobility and balance [18]. Starting from a sitting position in the middle of a chair with arm rests, participants were instructed to rise from the chair, walk $3 \mathrm{~m}$, turn around, walk back to the chair, and sit down at their regular pace. The time(s) between standing from the chair and sitting down again was recorded.

\section{Statistical analysis}

Data are expressed as mean \pm standard deviation. Clinical characteristics were compared between the control and LSS groups using Student's t tests. Analysis of covariance was used to determine the differences in physical fitness test results and ODI and QOL scores between the two groups after adjusting for age and BMI. Pearson's partial correlation analyses were performed to assess the relationships between the physical fitness measures and ODI and QOL scores between the control and LSS groups after adjusting for age and BMI. Analyses were performed with SPSS ver. 18.0 (SPSS Inc., Chicago, IL, USA) and the level of statistical significance was set as $p<0.05$.

\section{Results}

\section{Comparison of basic characteristics between the con- trol and LSS groups}

Table 1 shows the basic characteristics of participants in the LSS and control groups. Body weight, BMI, and fat

Table 1. Comparison of basic characteristics between the control and LSS groups

\begin{tabular}{lccc} 
Variable & Control $(\mathrm{n}=154)$ & Study $(\mathrm{n}=38)$ & $p$-value \\
\hline Age $(\mathrm{yr})$ & $70.6 \pm 4.0$ & $70.6 \pm 4.7$ & 0.99 \\
\hline Height $(\mathrm{cm})$ & $152.7 \pm 4.8$ & $152.3 \pm 6.5$ & 0.73 \\
Weight $(\mathrm{kg})$ & $56.0 \pm 7.1$ & $59.3 \pm 8.3$ & 0.01 \\
Body mass index $\left(\mathrm{kg} / \mathrm{m}^{2}\right)$ & $24.1 \pm 2.8$ & $25.7 \pm 3.3$ & $<0.01$ \\
\hline Fat content $(\%)$ & $34.3 \pm 6.4$ & $37.2 \pm 6.4$ & 0.01 \\
Skeletal muscle mass $(\mathrm{kg})$ & $19.3 \pm 2.3$ & $19.5 \pm 2.5$ & 0.65 \\
\hline Waist circumference $(\mathrm{cm})$ & $87.4 \pm 8.3$ & $86.5 \pm 8.2$ & 0.56 \\
\hline
\end{tabular}

Values are presented as mean \pm standard deviation.

LSS, lumbar spinal stenosis.

a)Derived with Student's $t$ test.

Table 2. Comparison of physical fitness, disability, and QOL between the control and LSS groups

\begin{tabular}{|c|c|c|c|c|}
\hline Variable & Control (n=154) & Study $(\mathrm{n}=38)$ & $p$-value ${ }^{\text {a) }}$ & Adjusted $p$-value ${ }^{b)}$ \\
\hline \multicolumn{5}{|l|}{ Physical fitness tests } \\
\hline Handgrip strength (kg) & $21.7 \pm 3.9$ & $19.4 \pm 4.5$ & $<0.01$ & $<0.01$ \\
\hline Cross-arm crunch sit-up (repetitions/60 sec) & $6.4 \pm 8.9$ & $4.2 \pm 9.4$ & 0.35 & 0.65 \\
\hline Sit-and-reach $(\mathrm{cm})$ & $17.3 \pm 6.9$ & $14.0 \pm 7.9$ & 0.04 & 0.04 \\
\hline Prone trunk extension (cm) & $24.3 \pm 8.3$ & $22.5 \pm 9.1$ & 0.38 & 0.11 \\
\hline Prone isometric chest raise (sec) & $46.2 \pm 34.2$ & $41.8 \pm 37.6$ & 0.67 & 0.94 \\
\hline Sit-to-stand (repetitions/30 sec) & $17.1 \pm 6.0$ & $11.6 \pm 3.3$ & $<0.01$ & $<0.01$ \\
\hline Functional reach $(\mathrm{cm})$ & $29.2 \pm 6.1$ & $25.9 \pm 8.1$ & 0.05 & 0.03 \\
\hline Timed up and go (sec) & $6.9 \pm 2.0$ & $8.3 \pm 2.2$ & $<0.01$ & $<0.01$ \\
\hline ODI score $(0-50)$ & $13.6 \pm 12.9$ & $32.8 \pm 15.3$ & $<0.01$ & $<0.01$ \\
\hline EQ-5D-5L index & $0.77 \pm 0.15$ & $0.57 \pm 0.13$ & $<0.01$ & $<0.01$ \\
\hline
\end{tabular}

Values are presented as mean \pm standard deviation.

QOL, quality of life; LSS, lumbar spinal stenosis; ODI, Oswestry disability index; EO-5D-5L, Euro0ol five-dimensional questionnaire.

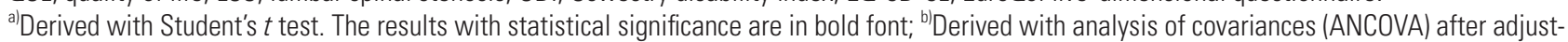
ing for age and body mass index. 
content were significantly higher in the LSS group than in the control group $(p<0.05)$. There were no differences in age, height, skeletal muscle mass, and waist circumference between the two groups.

\section{Comparison of physical fitness parameters, ODI, and QOL between the control and LSS groups}

After adjusting for age and BMI, handgrip strength (21.7 \pm 3.9 vs. $19.4 \pm 4.5, p<0.01)$, sit-and-reach test $(17.3 \pm$ 6.9 vs. $14.0 \pm 7.9, p=0.04)$, STS $(17.1 \pm 6.0$ vs. $11.6 \pm 3.3$, $p<0.01)$, functional reach test $(29.2 \pm 6.1$ vs. $25.9 \pm 8.1$, $p=0.03$ ), and TUG test results ( $6.9 \pm 2.0$ vs. $8.3 \pm 2.2, p<0.01$ ) were significantly higher in the control group than in the LSS group (Table 2). ODI scores were significantly higher and EQ-5D-5L scores were significantly lower in the LSS group than in the control group after adjusting for age and BMI, highlighting that participants with LSS had a greater degree of back pain-related disability and lower QOL.

\section{Association of physical fitness with functional dis- ability and QOL}

In the control group, the prone trunk extension test $(r=-0.21, p=0.02)$, prone isometric chest raise test $(r=-0.21$, $p<0.01)$, functional reach test $(r=-0.25, p<0.01)$, and TUG results $(r=0.22, p<0.01)$ were significantly correlated with both ODI and EQ-5D-5L scores after adjusting for age and BMI (Table 3). Handgrip strength was significantly correlated with EQ-5D-5L scores but not with ODI scores.

Table 3. Association of physical fitness with functional disability and $\mathrm{QOL}$

\begin{tabular}{|c|c|c|}
\hline Physical fitness tests ${ }^{a)}$ & ODI & EQ-5D-5L score \\
\hline \multicolumn{3}{|l|}{ Handgrip strength (kg) } \\
\hline Control & $-0.76(0.35)^{b)}$ & $0.23(<0.01)^{c}$ \\
\hline Study & $-0.37(0.03)^{c)}$ & $0.39(0.02)^{c)}$ \\
\hline \multicolumn{3}{|c|}{ Cross-arm crunch sit-up (repetitions/60 sec) } \\
\hline Control & $-0.21(0.81)$ & $0.08(0.31)$ \\
\hline Study & $-0.01(0.99)$ & $0.30(0.29)$ \\
\hline \multicolumn{3}{|l|}{ Sit-and-reach (cm) } \\
\hline Control & $0.75(0.36)$ & $0.06(0.41)$ \\
\hline Study & $-0.29(0.21)$ & $0.26(0.26)$ \\
\hline \multicolumn{3}{|l|}{ Prone trunk extension $(\mathrm{cm})$} \\
\hline Control & $-0.21(0.02)^{c)}$ & $0.18(0.02)^{c)}$ \\
\hline Study & $-0.27(0.27)$ & $0.39(0.12)$ \\
\hline \multicolumn{3}{|c|}{ Prone isometric chest raise (sec) } \\
\hline Control & $-0.21(<0.01)^{c}$ & $0.30(<0.00)^{c}$ \\
\hline Study & $-0.22(0.47)$ & $0.49(0.09)$ \\
\hline \multicolumn{3}{|c|}{ Sit-to-stand (repetitions/30 sec) } \\
\hline Control & $-0.10(0.24)$ & $0.18(0.02)^{c}$ \\
\hline Study & $-0.18(0.45)$ & $0.16(0.50)$ \\
\hline \multicolumn{3}{|l|}{ Functional reach $(\mathrm{cm})$} \\
\hline Control & $-0.25(<0.01)^{c}$ & $0.31(<0.01)^{c}$ \\
\hline Study & $-0.54(<0.01)^{c)}$ & $0.28(0.16)$ \\
\hline \multicolumn{3}{|l|}{ Timed up and go (sec) } \\
\hline Control & $0.22(<0.01)^{c}$ & $-0.30(<0.01)^{c}$ \\
\hline Study & $0.54(<0.01)^{c)}$ & $-0.32(0.08)$ \\
\hline
\end{tabular}

OOL, quality of life; ODI, Oswestry disability index; EQ-5D-5L, Euro0ol five-dimensional questionnaire.

alData are derived with partial correlation analysis between physical fitness tests and ODI, EQ-5D-5L after adjusting for age and body-mass index;

${ }^{b}$ Values are presented as $r$-value ( $p$-value) ( $r$, correlation coefficient; $p$-value, two-tailed); ${ }^{c}$ The results with statistical significance. 
In the LSS group, TUG test results were significantly correlated with ODI scores and handgrip strength was significantly correlated with both ODI and EQ-5D-5L scores.

\section{Discussion}

Our cross-sectional study demonstrated that some physical fitness test results (handgrip strength, sit-and-reach, STS, functional reach, and TUG) were significantly lower in the LSS group than in the control group after adjusting for age and BMI. Furthermore, handgrip strength, functional reach, and TUG test results were significantly associated with back pain-related disability and QOL in the LSS group.

Our findings of the differences between the LSS and control groups in the physical fitness tests are consistent with those of a previous study. Kim et al. [18] reported that patients with LSS showed significantly lower TUG and STS test results. Walking disturbance and standing intolerance in conjunction with pain are common symptoms among patients with LSS. Generalized stability, which we measured with the functional reach test, is also diminished by LSS. Furthermore, the results of the sit-and-reach test were lower in the LSS group than the control group, indicating decreased flexibility in the LSS group. Therefore, the distinction in statistical significance in the comparisons between the LSS and control groups may be explained by the symptoms and pathophysiology of spinal stenosis.

In our study, the results of handgrip strength, functional reach, and TUG tests in the LSS group were significantly related to ODI, but only handgrip strength was related to QOL. In LSS, back pain is known to lead to disturbance in standing and walking, which ultimately results in disability. As the functional reach test reflects stability and the TUG test reflects mobility and balance, it is possible that participants with more severe problems with walking and stability will complain of a higher degree of disability related to back pain. However, in contrast to other tests, handgrip strength is not directly dependent on the symptoms of spinal stenosis, but rather reflects generalized muscle strength. Therefore, our results suggest a possible association between generalized muscle weakness and the pathogenesis of LSS. Handgrip strength was the only factor that was correlated with both ODI and QOL in the LSS group. A comprehensive understanding of the mechanism that explains the relationships among hand- grip strength, back pain, and QOL is yet to be reached. However, we suggest a possible hypothesis based on our findings. First, enhanced basal muscle tone may reduce peripheral sensitization and pain [20]. A previous study reported that reduced basal muscle tone was associated with reduced resting state functional connectivity of sensory-motor systems and increased peripheral pain sensitization [5]. Therefore, increased basal muscle tone of the whole body induced by greater muscle strength may reduce peripheral pain sensitization, consequently reducing the degree of disability associated with back pain and improving QOL. Second, proinflammatory cytokines may be involved; increased proinflammatory cytokines are related to decreased muscle strength associated with age [21]. Increased oxidative stress, along with proinflammatory cytokines, induces the dysfunction of mitochondria and alters the synthesis of proteins, finally resulting in the loss of muscle function [22]. Therefore, although it is impossible to attribute causality with the results of our cross-sectional study, increased levels of proinflammatory cytokines with age may mediate the relationship of muscle strength to the degree of disability related to back pain and the decline of QOL in patients with LSS.

Our study has some limitations. First, the cross-sectional design could not determine any causality. Second, only a small number of women were assigned to the LSS group, making it is impossible to generalize our results. Third, we defined the LSS group based on simple radiography findings and subjective symptoms. Finally, we did not measure appendicular muscle mass, which reflects the muscle mass of the body, but only measured muscle strength.

\section{Conclusions}

In conclusion, elderly women with LSS showed significantly lower physical fitness than the control group. Some physical fitness test results were also associated with the degree of disability related to back pain and QOL in the LSS group. In particular, handgrip strength (which reflects generalized muscle strength and is not considered to be influenced by LSS symptoms) was significantly lower in the LSS group than in the control group, and was correlated with the degree of disability and QOL in the LSS group. Although we could not determine causality, our results collectively suggest that enhancing muscle strength reduces the degree of disability due to back pain and improves QOL in patients with LSS. Further prospective, 
large-scale studies are required to verify our findings.

\section{Conflict of Interest}

No potential conflict of interest relevant to this article was reported.

\section{Acknowledgements}

This study was supported by the National Health Promotion Fund, 2014.

\section{ORCID}

Saejong Park: 0000-0001-7229-5790

Ho Sung Han: 0000-0001-5080-3862

Gang-Un Kim: 0000-0001-9772-4977

Sung Shik Kang: 0000-0001-8914-8375

Ho-Joong Kim: 0000-0002-8205-4648

Mihyun Lee: 0000-0002-3827-3405

Soo Hyun Park: 0000-0003-4808-0639

Kyu Hwan Choi: 0000-0002-6636-0653

Sung-Ho Kim: 0000-0001-9213-8362

Jin S. Yeom: 0000-0002-8241-5335

\section{References}

1. Lee SY, Kim TH, Oh JK, Lee SJ, Park MS. Lumbar stenosis: a recent update by review of literature. Asian Spine J 2015;9:818-28.

2. Ishimoto Y, Yoshimura N, Muraki S, et al. Prevalence of symptomatic lumbar spinal stenosis and its association with physical performance in a populationbased cohort in Japan: the Wakayama Spine Study. Osteoarthritis Cartilage 2012;20:1103-8.

3. Rampersaud YR, Ravi B, Lewis SJ, et al. Assessment of health-related quality of life after surgical treatment of focal symptomatic spinal stenosis compared with osteoarthritis of the hip or knee. Spine J 2008;8: 296-304.

4. Genevay S, Atlas SJ. Lumbar spinal stenosis. Best Pract Res Clin Rheumatol 2010;24:253-65.

5. Soriano-Maldonado A, Ruiz JR, Aparicio VA, et al. Association of physical fitness with pain in women with fibromyalgia: the al-Andalus Project. Arthritis Care Res (Hoboken) 2015;67:1561-70.

6. Juraschek SP, Blaha MJ, Whelton SP, et al. Physical fitness and hypertension in a population at risk for cardiovascular disease: the Henry Ford ExercIse Testing (FIT) Project. J Am Heart Assoc 2014;3:e001268.

7. Alosco ML, Spitznagel MB, Cohen R, et al. Obesity and cognitive dysfunction in heart failure: the role of hypertension, type 2 diabetes, and physical fitness. Eur J Cardiovasc Nurs 2015;14:334-41.

8. Bootsma-van der Wiel A, Gussekloo J, De Craen AJ, Van Exel E, Bloem BR, Westendorp RG. Common chronic diseases and general impairments as determinants of walking disability in the oldest-old population. J Am Geriatr Soc 2002;50:1405-10.

9. Suzuki R, Rygh LJ, Dickenson AH. Bad news from the brain: descending 5-HT pathways that control spinal pain processing. Trends Pharmacol Sci 2004; 25:613-7.

10. Kapoor M, Martel-Pelletier J, Lajeunesse D, Pelletier JP, Fahmi H. Role of proinflammatory cytokines in the pathophysiology of osteoarthritis. Nat Rev Rheumatol 2011;7:33-42.

11. Kreiner DS, Shaffer WO, Baisden JL, et al. An evidence-based clinical guideline for the diagnosis and treatment of degenerative lumbar spinal stenosis (update). Spine J 2013;13:734-43.

12. Schizas C, Theumann N, Burn A, et al. Qualitative grading of severity of lumbar spinal stenosis based on the morphology of the dural sac on magnetic resonance images. Spine (Phila Pa 1976) 2010;35:191924.

13. Jensky-Squires NE, Dieli-Conwright CM, Rossuello A, Erceg DN, McCauley S, Schroeder ET. Validity and reliability of body composition analysers in children and adults. Br J Nutr 2008;100:859-65.

14. Jeon CH, Kim DJ, Kim SK, Lee HM, Park HJ. Validation in the cross-cultural adaptation of the Korean version of the Oswestry Disability Index. J Korean Med Sci 2006;21:1092-7.

15. Hwang HJ, Kim SH. The association among three aspects of physical fitness and metabolic syndrome in a Korean elderly population. Diabetol Metab Syndr 2015;7:112.

16. del Pozo-Cruz B, Gusi N, Adsuar JC, del Pozo-Cruz J, Parraca JA, Hernandez-Mocholi M. Musculoskeletal fitness and health-related quality of life characteristics among sedentary office workers affected by subacute, non-specific low back pain: a cross-sectional study. Physiotherapy 2013;99:194-200. 
17. Moreau CE, Green BN, Johnson CD, Moreau SR. Isometric back extension endurance tests: a review of the literature. J Manipulative Physiol Ther 2001;24: 110-22.

18. Kim HJ, Chun HJ, Han CD, et al. The risk assessment of a fall in patients with lumbar spinal stenosis. Spine (Phila Pa 1976) 2011;36:E588-92.

19. Weiner DK, Duncan PW, Chandler J, Studenski SA. Functional reach: a marker of physical frailty. J Am Geriatr Soc 1992;40:203-7.
20. Hirakawa N, Tershner SA, Fields HL, Manning BH. Bi-directional changes in affective state elicited by manipulation of medullary pain-modulatory circuitry. Neuroscience 2000;100:861-71.

21. Doria E, Buonocore D, Focarelli A, Marzatico F. Relationship between human aging muscle and oxidative system pathway. Oxid Med Cell Longev 2012; 2012:830257.

22. Drey M. Sarcopenia: pathophysiology and clinical relevance. Wien Med Wochenschr 2011;161:402-8. 